\title{
Selecting a contingency table in a population-based association study: allele frequency or positivity?
}

Received: January 5, 1999 / Accepted: February 16, 1999

\begin{abstract}
In population-based association studies, the significance of the association between a candidate gene and a disease is usually examined by a simple $\chi^{2}$ test. Such studies require a $2 \times 2$ contingency table made up of either allele frequencies or positivities in affected and control groups. In order to investigate the influence of each $2 \times 2$ table on the power of the $\chi^{2}$ test, $P$ values were calculated for two penetrance models (multiplicative and additive). When the value of penetrance was small and not markedly different among genotypes, a large difference in the power of the $\chi^{2}$ test was observed between the two tables. In a multiplicative model, the allele frequency table was superior to the positivity table for detecting significance. In contrast, in an additive model, the positivity table was most suitable. Selecting a contingency table was especially important for detecting true association, when the required significance level was corrected to avoid the problem of multiple hypothesis testing. The $\chi^{2}$ test, therefore, should be performed for both tables to identify a susceptible gene with a low penetrance, even when no significant difference is observed in one table.
\end{abstract}

Key words Association study $\cdot \chi^{2}$ test $\cdot 2 \times 2$ Contingency table $\cdot$ Allele frequency $\cdot$ Allele positivity

\section{Introduction}

With progress in human genome analyses, a large number of human genes have been identified, and genes causing most single-gene diseases should be identified in the near future. The next important field in human genetics is the elucidation of gene variations among individuals and their associations with polygenic disorders. Currently, many geneticists are in-

J. Ohashi $(\bowtie) \cdot K$. Tokunaga

Department of Human Genetics, School of International Health, Graduate School of Medical Sciences, University of Tokyo, 7-3-1

Hongo, Bunkyo-ku, Tokyo 113-0033, Japan

Tel. +81-3-3812-2111 (ext. 3483, 3693); Fax +81-3-5802-8619

e-mail: juno@.m.u-tokyo.ac.jp volved in the genetic dissection of complex traits which do not show simple Mendelian monogenic inheritance (Lander and Schork 1994). The population-based association study, which tests the hypothesis that variant(s) of a particular gene contribute to the pathogenesis of a certain disease is one powerful approach. In such studies, a $\chi^{2}$ test is carried out with a $2 \times 2$ contingency table made up of either the number of a particular allele (i.e. allele frequency table) or the number of individuals possessing a particular allele (i.e. positivity table) in affected and control individuals. Although the power of the $\chi^{2}$ test is affected by the type of $2 \times 2$ table (allele frequency or positivity), it remains unclear which type of table is more appropriate for detecting a susceptible gene in a multifactorial disease with a low penetrance. The main purpose of this study was to examine the influence of these factors on the power of the $\chi^{2}$ test.

\section{Model}

Let us consider a large random mating population in which several alleles exist at the candidate locus for a certain disease susceptibility. We assume that allele $A$, the frequency of which is $p$, is a susceptible allele at this locus. The other alleles are called allele $a$ for the sake of convenience. Let $f_{A A}, f_{A a}$, and $f_{a a}$ be the penetrances determined by genotypes $A A, A a$, and $a a$, respectively. Noting that the HardyWeinberg equilibrium is satisfied in the whole population, genotype frequencies of $A A, A a$, and $a a$ are $p^{2} f_{A A} / e, 2 p(1-$ $p) f_{A a} / e$, and $(1-p)^{2} f_{a a} / e$, respectively, in the affected group, where $e$ denotes the disease prevalence (i.e., $e=p^{2} f_{A A}+$ $\left.2 p(1-p) f_{A a}+(1-p)^{2} f_{a a}\right)$. In the control group, genotype frequencies of $A A, A a$, and $a a$ are $p^{2}\left(1-f_{A A}\right) /(1-\mathrm{e}), 2 p(1$ $-p)\left(1-f_{A a}\right) /(1-\mathrm{e})$, and $(1-p)^{2}\left(1-f_{a a}\right) /(1-\mathrm{e})$. When $N$ and $M$ individual samples are obtained from affected and control groups, respectively, the expected number of copies of allele $A$ in the affected group is given by $2 N \times\left(p^{2} f_{A A}+\right.$ $\left.p(1-p) f_{A a}\right) / e$. The expected number of individuals possessing allele $A$ in the affected group becomes $N \times\left(p^{2} f_{A A}+\right.$ $\left.2 p(1-p) f_{A a}\right) / e$. Likewise, those numbers in the control 
group are given by $2 M \times\left(p^{2}\left(1-f_{A A}\right)+p(1-p)\left(1-f_{A a}\right)\right) /$ $(1-\mathrm{e})$ and $M \times\left(p^{2}\left(1-f_{A A}\right)+2 p(1-p)\left(1-f_{A a}\right)\right) /(1-\mathrm{e})$, respectively. On the basis of the above calculations, $2 \times 2$ contingency tables were made, and the $\chi^{2}$ value was then calculated for each case.

\section{Results and discussion}

In the present study, two models of inheritance, multiplicative (i.e., $f_{A A}=r^{2} \times f_{a a}, f_{A a}=r \times f_{a a}$; where $r=$ is a risk parameter for multiplicative model) and additive (i.e., $f_{A A}=$ $2 d+f_{a a}, f_{A a}=d+f_{a a}$; where $d=$ risk parameter for additive model), were examined for the case that $N=M=50$. Figure 1 shows the relationship between the frequencies of the susceptible allele and the $P$ values in the multiplicative model $(r=3)$. Penetrances of $f_{A A}, f_{A a}$, and $f_{a a}$ were assumed to be $0.09,0.03$, and 0.01 , respectively. The allele frequency table was superior to the positivity table for detecting significance.

Figure 2 shows the results from the additive model $(d=$ 0.04 ) in which $f_{A A}, f_{A a}$, and $f_{a a}$ were assumed to be $0.09,0.05$, and 0.01 , respectively. In contrast to Fig. 1 , the positivity table was suitable for detecting the susceptible allele when its frequency was less than 0.42 . The difference between two inheritance models was the penetrance of the $A a$ genotype, and it was very slight ( 0.03 and 0.05$)$. Nevertheless, results were quite different from each other. The present finding that the allele frequency plays a significant role in determining the usefulness of the $\chi^{2}$ test can predict the following case: a positive significant association is detected by the first study, yet this association is not confirmed in the second study, not because of a sampling error or some additional genetic or environmental factor(s), but simply because of

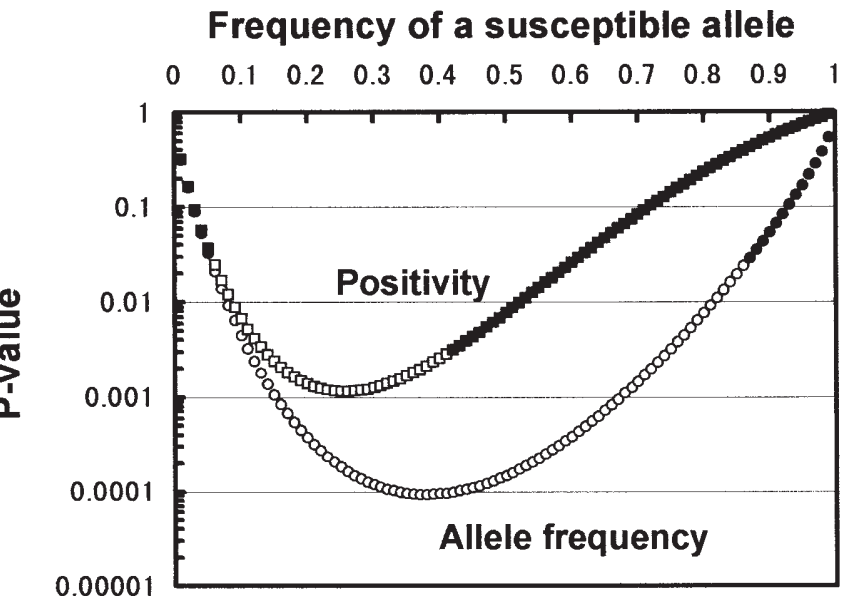

Fig. 1. $P$ values expected from $\chi^{2}$ tests for $N$ (individual samples from affected group) $=M$ (individual samples from control group) $=50$. Results were given by each table: the allele frequency table (indicated by open circles and closed circles) and the positivity table (indicated by open squares and closed squares). Closed circles and closed squares represent cases in which the expected number in one of the cells of a $2 \times 2$ table was less than 5. Penetrances were assumed that $f_{A A}=0.09$, $f_{A a}=0.03$, and $f_{a a}=0.01$ (multiplicative model, $r=3$ ). See text for definitions of $f_{A A}, f_{A a}$, and $f_{a a}$; and $r$ the difference in frequencies of the candidate alleles in the studied populations. Therefore, the detection of a significant association could sometimes depend on the frequency of the candidate allele in a subject population, although we have usually overlooked this possibility. The sample size was critical to the power of the $\chi^{2}$ test, while the selection of a proper $2 \times 2$ table was not influenced by the sample size in most cases (data not shown).

Let us consider an actual example. Multiple sclerosis has been reported to be associated with the HLA serotype. On the assumption that the population prevalence was $0.1 \%$, penetrances for the genotypes DR2/DR2, DR2/DRx, and DRx/DRx were estimated to be 0.002874, 0.002691, and 0.000442 , respectively (Risch 1987). In this case, the positivity table was found to be more suitable than the allele frequency table for detecting the association (Fig. 3). Since there is a problem of multiple hypothesis testing for such a multiallelic gene as HLA, if $n$ alleles exist at the locus, the required significance level should be divided by $n-1$. Therefore, as the number of tested alleles increases, the selection of a proper table becomes very important.

In the case of a multifactorial or complex disease, the penetrance is usually low even for individuals homozygous for the susceptible allele, because other genes and environmental factors also contribute to the onset of the disease. In addition, the affected group usually contains some individuals with non-susceptible allele(s). As described above, it is difficult to predict which table would be more appropriate. Therefore, the $\chi^{2}$ test should be performed for both the allele frequency and the positivity tables, even when no significant difference is observed in one table. This leads, however, to the carrying out of independent $\chi^{2}$ tests twice for the same allele. To avoid such a problem of multiple testing, the required significance level may be divided by two. When the difference is significant in one table but not

\section{Frequency of a susceptible allele}
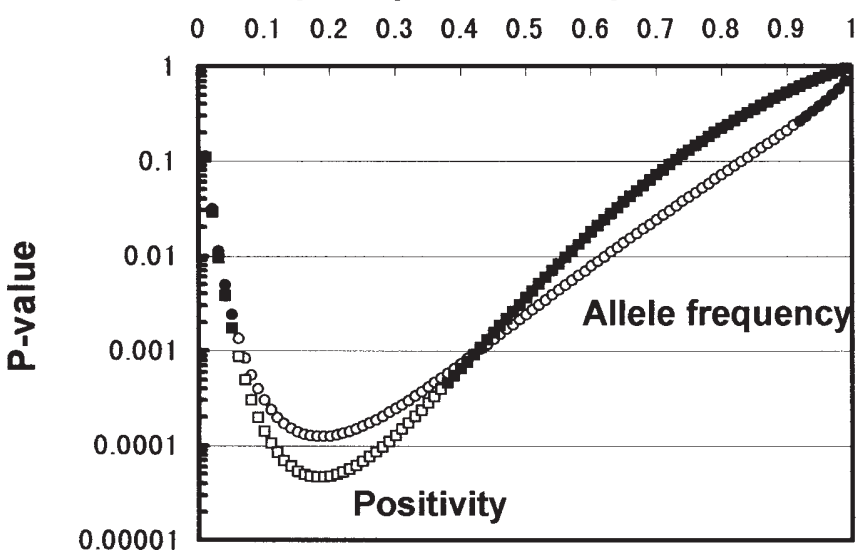

Fig. 2. $P$ values expected from $\chi^{2}$ tests for $N=M=50$. Results were given by each table: the allele frequency table (indicated by open circles and closed circles) and the positivity table (indicated by open squares and closed squares). Closed circles and closed squares represent cases in which the expected number in one of the cells of a $2 \times 2$ table was less than 5. Penetrances were assumed that $f_{A A}=0.09, f_{A a}=0.05$, and $f_{a a}=$ 0.01 (additive model, $d=0.04$ ). See text for definition of $d$ 
Frequency of DR2

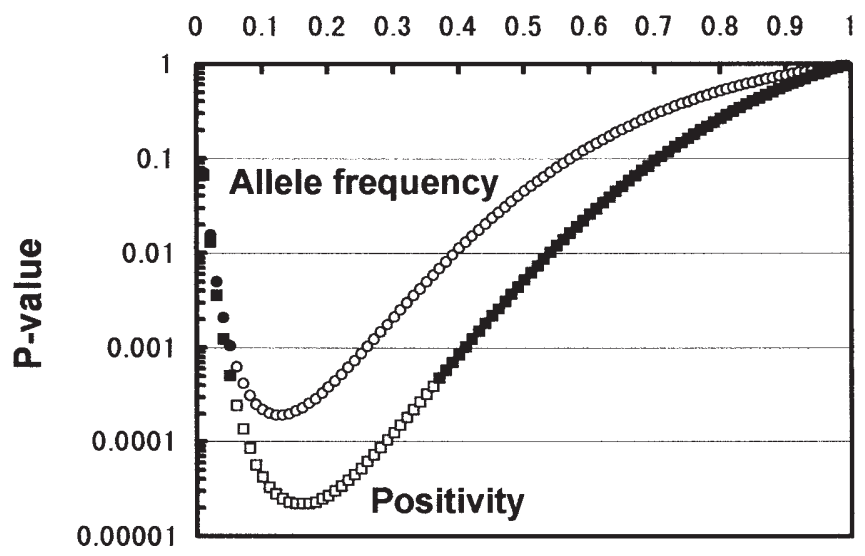

Fig. 3. $P$ values expected from $\chi^{2}$ tests for detecting the association between multiple sclerosis and the HLA allele DR2. Results were given by each table: the allele frequency table (indicated by open circles and closed circles) and the positivity table (indicated by open squares and closed squares). Penetrances for the genotypes DR2/DR2, DR2/ DRx, and DRx/DRx were determined to be $0.002874,0.002691$, and 0.000442 , respectively (Risch 1987). It was assumed that $N=M=50$

in the other, we should estimate the penetrance from the actual data and then examine whether the discordance can be explained by the present model. For example, if $f_{A a}$ is estimated to be greater than $f_{A A}$, the apparent association may be caused by a sampling error.

The statistical problems of population-based association studies have been pointed out previously, with major attention paid to the selection of suitable controls (Lander and Schork 1994). Recently, to avoid the occurrence of falsepositive results caused by population stratification, the transmission disequilibrium test (TDT), which is an association study with internal controls, was proposed (Spielman et al. 1993). However, this family-based test suffers from the drawback that parent samples have to be available for the analysis, a requirement which is unlikely to be fulfilled for late-onset diseases (Strachan and Read 1996). Although the sib TDT (S-TDT), which is based on data from affected and unaffected offspring, was developed from the TDT for use when the parental genotype cannot be obtained (Spielman and Ewens 1998), neither the TDT nor the S-TDT is suitable for testing rare alleles or susceptible alleles with low penetrances because it is difficult to obtain data from sufficient numbers of family members. Accordingly, a population-based association study is still required and is essential for the identification of such susceptible genes with low penetrances in complex diseases.

Acknowledgments This work was supported by Grants-in-Aid for Scientific Research from the Ministry of Education, Science, and Culture of Japan and a grant from the Uehara Memorial Foundation.

\section{References}

Lander ES, Schork NJ (1994) Genetic dissection of complex traits. Science 265:2037-2048

Risch N (1987) Assessing the role of HLA-linked and unlinked determinants of disease. Am J Hum Genet 40:1-14

Spielman RS, McGinnis RE, Ewens WJ (1993) Transmission test for linkage disequilibrium: The insulin gene region and insulindependent diabetes mellitus (IDDM). Am J Hum Genet 52:506-516

Spielman RS, Ewens WJ (1998) A sibship test for linkage in the presence of association: the sib transmission/disequilibrium test. Am J Hum Genet 62:450-458

Strachan T, Read AP (1996) Human molecular genetics, 1st edn. John Wiley and Sons, New York 